Document downloaded from:

http://hdl.handle.net/10251/67488

This paper must be cited as:

Palma, R.; Pérez-Aparicio, JL.; Taylor, R. (2012). Non-linear finite element formulation applied to thermoelectric materials under hyperbolic heat conduction model. Computer Methods in Applied Mechanics and Engineering. 213-216:93-103.

doi:10.1016/j.cma.2011.11.011.

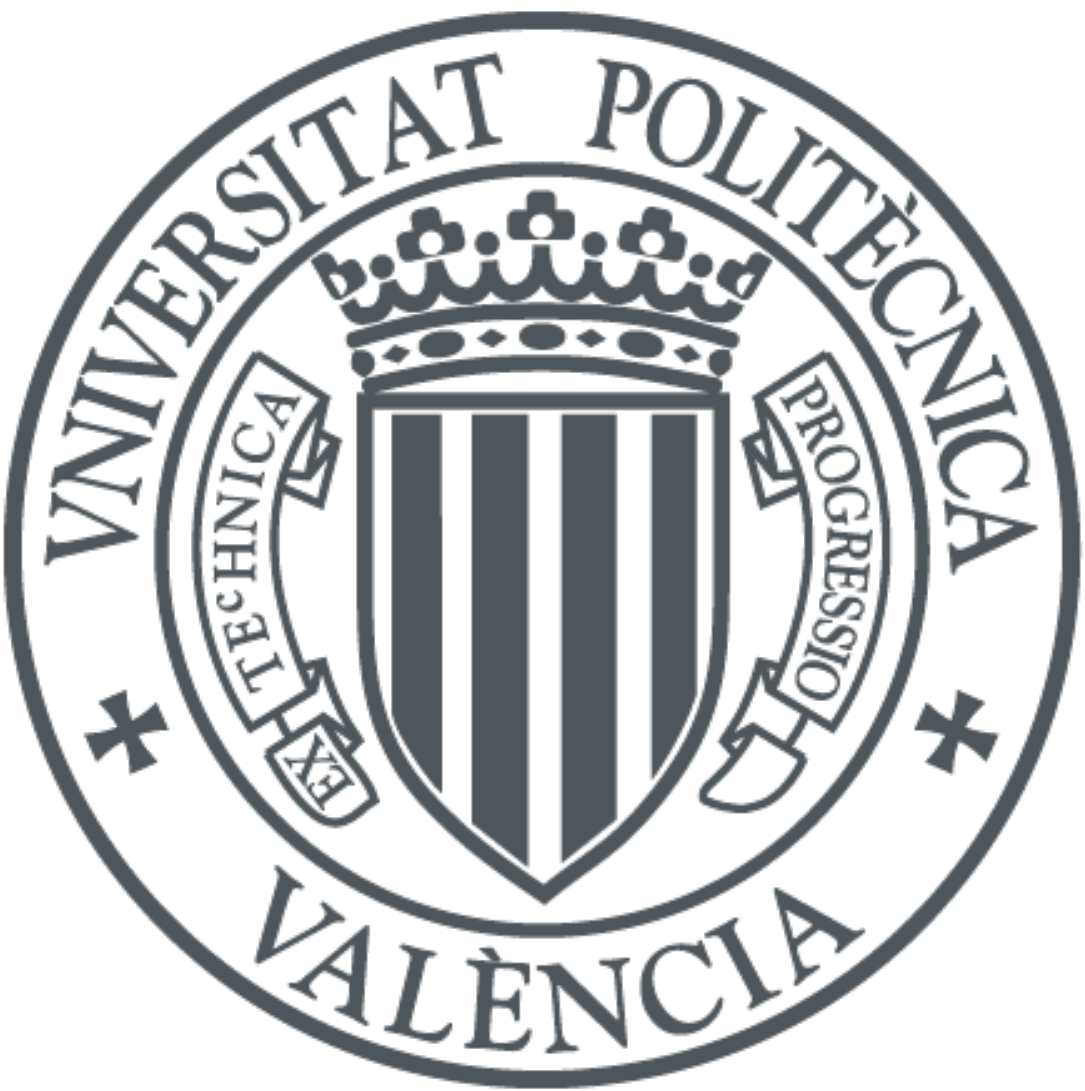

The final publication is available at

http://dx.doi.org/10.1016/j.cma.2011.11.011

Copyright Elsevier

Additional Information 


\title{
Non-linear Finite Element Formulation Applied to Thermoelectric Materials under Hyperbolic Heat Conduction Model
}

\author{
R. Palma ${ }^{\mathrm{a}}$, J.L. Pérez-Aparicio ${ }^{\mathrm{a}}$, R.L. Taylor ${ }^{\mathrm{b}}$ \\ ${ }^{a}$ Mecánica de Medios Continuos y Teoría de Estructuras, Universidad Politécnica de Valencia, Spain \\ ${ }^{b}$ Department of Civil and Environmental Engineering, University of California at Berkeley, California, USA
}

\begin{abstract}
In the present work, a 3-D, dynamic and non-linear finite element to simulate thermoelectric behavior under a hyperbolic heat conduction model is presented. The transport equations, which couple electric and thermal energies by the Seebeck, Peltier and Thomson effects, are analytically obtained through extended non-equilibrium thermodynamics, since the local equilibrium hypothesis is not valid under the hyperbolic model. In addition, 1-D analytical solutions are obtained to validate the finite element formulation. Numerically, standard isoparametric eight-node elements with two degrees of freedom (voltage and temperature) per node are used. Non-linearities due to the temperature-dependence on the transport properties and the Joule effects are addressed with the Newton-Raphson algorithm. For the dynamic problem, HHT and Newmark- $\beta$ algorithms are compared to obtain accurate results, since numerical oscillations (Gibbs phenomena) are present when the initial boundary conditions are discontinuous. The last algorithm, which is regularized by relating time steps and element sizes, provides the best results. Finally, the finite element implementation is validated comparing the analytical and the numerical solutions.
\end{abstract}

Keywords:

Thermoelectrics, Dynamics, Non-linear Finite Element, Second sound, HHT algorithm, Newmark- $\beta$ algorithm

\section{Introduction}

Thermoelectric materials couple electric and thermal energies by means of three separated transport effects: Seebeck, Peltier and Thomson. In addition, the Ohm and Fourier laws that are inherent to electric and thermal fluxes are also present. Thermoelectric materials are used as heat pumps (heating and cooling) and generators, see [1]. In the last decade, miniaturized thermoelectric devices and high-frequency processes are increasingly applied to cool micro-electronic devices, see [2].

The classical Fourier law leads to a parabolic heat propagation problem that is incorrect from a physical point of view: especially in micro-devices and under rapid transient effects such as micro-pulses. Cattaneo [3] proposed a modification to the Fourier law that leads to a hyperbolic heat propagation problem. The modification is made through the introduction of a relaxation time $\tau$ that is an empirical parameter defined as the time-interval between two successive collisions at the microscopic level of either holes or electrons.

From a theoretical point of view, classical non-equilibrium thermodynamics [4] cannot lead to hyperbolic propagation since the local equilibrium hypothesis is not valid. For this reason, extended non-equilibrium thermodynamics [4], [5] must be applied to obtain the correct transport equations.

For heat propagation (without thermoelectric coupling) under the Cattaneo model analytical investigations were performed using the Laplace transform technique in [6], [7] and [8]. In [9] a mixed Finite Element (FE) formulation using the Crank-Nicolson scheme for time-integration was presented and in [10] a finite difference scheme was developed to compare implicit and explicit Euler algorithms. Several FE works in which the Cattaneo model was assumed also have been published. For example, in [11] and [12] FE's were formulated to simulate convection-diffusion (Fick law) and thermomechanical coupling, respectively. For thermoelectrics, in [13] the Laplace transform technique was used to solve problems formulated using the hyperbolic model. There are several FE formulations applied to the thermoelectric problem with the Fourier (parabolic) model, see [14] and [15].

The aim of the current work is to develop a threedimensional, transient, non-linear FE formulation to solve general thermoelectric problems with the hyperbolic model. For this purpose, a non-equilibrium entropy depending on temperature and heat flux is defined and the transport equations are formulated by applying the Onsager relations. Transport and balance equations are then transformed into a matrix form and implemented into the research FE code FEAP, [16]. The non-linearities due to: (i) Joule effect, (ii) temperature dependence of transport properties and (iii) thermo-electric coupling, are solved with the Newton-Raphson algorithm. Two discrete algorithms for transient solutions, namely, HHT or $\alpha$ [17] and Newmark- $\beta(\mathrm{N}-\beta)$ are compared. Both are regularized by means of linear relationship between time steps and element lengths. In order to validate the FE behavior, four 1$\mathrm{D}$ analytical solutions are compared with the numerical results. When the initial boundary conditions are discontinuous, strong oscillations (Gibbs phenomena) are observed and the $\mathrm{N}-\beta$ algorithm, with modified $\bar{\beta}$ and $\bar{\gamma}$ parameters, provides results that 
are consistent and accurate with respect to the analytical solutions.

\section{Thermodynamic formulation}

The aim of this section is to obtain balance and transport equations for the hyperbolic thermoelectric problem. As mentioned above, the hyperbolic problem cannot be obtained from classical non-equilibrium thermodynamics. This is due to the lack of validity of the local equilibrium hypothesis that states the equality of the local relations between thermodynamic quantities in a system in and out of equilibrium, [5]. The validity of this hypothesis is closely related with the Deborah number $D e=\tau / \tau_{M}<1$, where $\tau_{M}$ is the macroscopic experiment or effect duration. For materials such as polymers or under high-frequency effects such as ultrasounds, the time scale is comparable to $\tau, D e \geq 1$ and extended non-equilibrium thermodynamics need to be used.

There are several analytical procedures such as rational [18] or extended non-equilibrium thermodynamics [5] to study the transport equations when $D e \geq 1$. The former procedure is axiomatic, therefore in the present work the latter is used and the transport equations are obtained using a physical point of view.

\subsection{Balance laws}

Consider a thermodynamic universe composed from a system of domain $\Omega$, boundary $\Gamma$ and its surroundings, see Figure 1. Assuming the validity of a continuum hypothesis, the balance laws of continuum physics are those of charge and energy. The first is obtained from a Maxwell equation, see [19]

$$
\dot{\rho}_{\Omega}=-\nabla \cdot \boldsymbol{j}
$$

where a dot (') denotes a time derivative, $\boldsymbol{j}$ is electric flux and $\rho_{\Omega}$ is charge density.

An energy balance is obtained by applying the first law of thermodynamics: $\rho \dot{u}=\dot{W}+\dot{Q}$, where $\rho$ is mass density and $\dot{u}, \dot{W}, \dot{Q}$ are rates of internal energy density, electromagnetic energy and exchanged heat, respectively.

The rate of electromagnetic energy is given by the Poynting theorem [19]

$$
\dot{W}=\boldsymbol{E} \cdot \dot{\boldsymbol{P}}+\boldsymbol{B} \cdot \dot{\mathbf{M}}+\boldsymbol{j} \cdot \boldsymbol{E}
$$

where $\boldsymbol{E}, \boldsymbol{P}, \boldsymbol{B}$ and $\mathbf{M}$ are the electric field, polarization, magnetic flux and magnetization, respectively. The first two terms on the right-hand side of (2) represent the reversible work performed by the electromagnetic forces. The last term is the irreversible work (Joule heating), that represents the rate of electromagnetic energy converted into thermal energy.

The rate of exchanged heat is given by

$$
\dot{Q}=-\nabla \cdot \boldsymbol{s}+Q_{\Omega}
$$

where $Q_{\Omega}$ is the heat source created inside $\Omega$ and $\boldsymbol{\varsigma}$ the heat flux.

In the current work, the following terms are assumed to be zero: (i) $\rho_{\Omega}, Q_{\Omega}$ which is valid for most thermoelectric applications [20], (ii) $\boldsymbol{P}$ implying that a thermoelectric material is not a polarizable material, (iii) $\mathbf{M}$ implying no magnetic effects. Therefore, the charge and energy balances are

$$
\nabla \cdot \boldsymbol{j}=0 ; \quad \rho \dot{u}=-\nabla \cdot \boldsymbol{s}+\boldsymbol{j} \cdot \boldsymbol{E}
$$

\subsection{Transport equations}

The extended non-equilibrium thermodynamics theory assumes the existence of a non-equilibrium entropy $s$, that depends on the state variables and the fluxes. This situation is called "mixed" in continuum mechanics. Applying the hyperbolic model only to the thermal field, $s$ depends on $u$ and $\boldsymbol{\varsigma}$ [4]

$$
\dot{s}(\dot{u}, \dot{\boldsymbol{\zeta}})=\frac{\dot{u}}{T}-\frac{\tau}{\rho \kappa T^{2}} \boldsymbol{\varsigma} \cdot \dot{\boldsymbol{\zeta}}
$$

where $T$ and $\kappa$ denote temperature and thermal conductivity, respectively. The entropy balance is obtained by solving for $\dot{u}$ in (5) and introducing the result into the energy balance equation (4)-right

$$
\rho \dot{s}+\nabla \cdot\left(\frac{\boldsymbol{\zeta}}{T}\right)=\frac{\boldsymbol{j} \cdot \boldsymbol{E}}{T}+\boldsymbol{\varsigma} \cdot\left[\nabla\left(\frac{1}{T}\right)-\frac{\tau}{\kappa T^{2}} \dot{\boldsymbol{\zeta}}\right]
$$

where the relation $\nabla \cdot \boldsymbol{\varsigma} / T=\nabla \cdot(\boldsymbol{\varsigma} / T)-\boldsymbol{\varsigma} \cdot \nabla(1 / T)$ has been used. The general form of the entropy balance is [4]

$$
\rho \dot{s}+\nabla \cdot \boldsymbol{j}_{s}=\sigma^{s}
$$

where $\boldsymbol{j}_{\boldsymbol{s}}$ and $\sigma^{s}$ denote the entropy flux and the rate of entropy production, respectively. To obtain these magnitudes for the thermoelectric problem (6) and (7) are compared

$$
\boldsymbol{j}_{s}=\frac{\boldsymbol{\varsigma}}{T} ; \quad \sigma^{s}=\frac{\boldsymbol{j} \cdot \boldsymbol{E}}{T}+\boldsymbol{\varsigma} \cdot\left[\nabla\left(\frac{1}{T}\right)-\frac{\tau}{\kappa T^{2}} \dot{\boldsymbol{\zeta}}\right]
$$

The classical form of the entropy production for the thermoelectric problem, see [21], is recovered if $\tau=0$ : the hyperbolic model adds a term to the entropy production.

The transport equations will be obtained by expressing the entropy production in the form [5]

$$
\sigma^{s}=\boldsymbol{j} \cdot \nabla V+\dot{\boldsymbol{\zeta}} \cdot \frac{\partial s}{\partial \boldsymbol{s}} \geq 0
$$

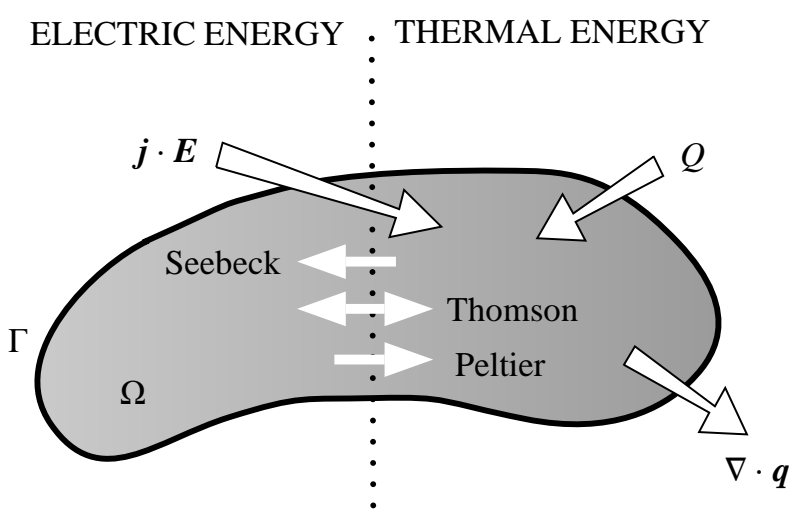

Figure 1: Energy balance: thermal flux, reversible and irreversible coupling. 
where $V$ is the voltage. With a sufficiently good approximation, fluxes and gradients are linearly related by phenomenological coefficients, which have to satisfy the Onsager-Casimir reciprocal relation. Finally, the transport equations are

$$
\begin{aligned}
& \boldsymbol{j}=-\gamma \nabla V-\alpha \gamma \nabla T \\
& \boldsymbol{\varsigma}+\tau \dot{\boldsymbol{S}}=-\kappa \nabla T+\alpha T \boldsymbol{j}
\end{aligned}
$$

where $\alpha, \gamma, \kappa$ denote the Seebeck coefficient, electric and thermal conductivities, respectively. These phenomenological coefficients are denominated thermoelectric properties in the following. Again, if $\tau=0$ in (10)-bottom the classical transport equations are restored.

Although linear relationships between fluxes and gradients have been used, the thermoelectric properties depend strongly on temperature (material non-linearity), accordingly in the present work

$$
\begin{aligned}
& \alpha(T)=1.98 \times 10^{-4}+3.35 \times 10^{-7} T-7.52 \times 10^{-10} T^{2} \\
& \gamma(T)=1.09 \times 10^{5}-5.59 \times 10^{2} T+2.49 T^{2} \\
& \kappa(T)=1.66-3.58 \times 10^{-3} T+3.19 \times 10^{-5} T^{2}
\end{aligned}
$$

in which dimensions are $\alpha[\mathrm{V} / \mathrm{K}], \gamma[\mathrm{A} / \mathrm{mV}], \kappa[\mathrm{W} / \mathrm{mK}]$ but the temperature is introduced in Celsius ${ }^{\circ} \mathrm{C}$ degrees, see [23].

\subsection{Strong form}

From the previous equations and taking into account the coupled nature of the thermoelectric problem, the strong form is formed as a system of two coupled partial differential equations. The electric strong form is obtained by replacing (10)top into (4)-left and considering the temperature-dependence of the thermoelectric properties (11)

$$
\begin{aligned}
& \gamma(T) \nabla^{2} V+\partial_{T}\{\gamma(T)\} \nabla T \cdot \nabla V= \\
& -\left[\gamma(T) \partial_{T}\{\alpha(T)\}+\alpha(T) \partial_{T}\{\gamma(T)\}+\alpha(T) \gamma(T)\right] \nabla^{2} T
\end{aligned}
$$

Here and in the following, $\partial_{T}$ implies partial derivation with respect to the subscript, in this equation the temperature $T$. In order to obtain analytical solutions, from (10)-top

$$
\nabla V=-\alpha(T) \nabla T-\frac{j}{\gamma(T)}
$$

where the first term in the right-hand represents the thermal energy converted into electric by the Seebeck effect. Due to restrictions in analytical solutions (not in the numerical ones), in this article we present cases in which only $\alpha$ is variable with $T$. The values of $\kappa, \gamma$ will be evaluated at a certain average temperature from (11).

The thermal strong form is obtained substituting (10)-bottom into (4)-right and using (4)-left. Furthermore, the relations $u=$ $c T$ (c, specific capacity), $\boldsymbol{E}=-\nabla V$ and (11) are introduced

$$
\begin{aligned}
& \tau \rho c \ddot{T}+\rho c \dot{T}= \\
& \qquad \nabla^{2} T+\frac{\boldsymbol{j}^{2}}{\gamma}-t_{h} \nabla T \cdot \boldsymbol{j}-\tau \partial_{t}\{\boldsymbol{j} \cdot \nabla V\}
\end{aligned}
$$

where $t_{h}=T[\partial \alpha(T) / \partial T]$ is the Thomson coefficient. This expression is an hyperbolic equation (notice the first term on the
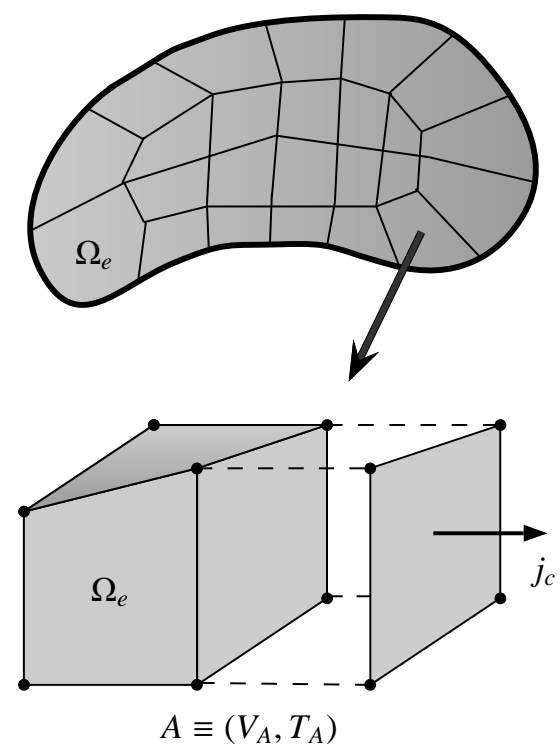

Figure 2: Domain discretization. 3-D and interface finite elements.

left-hand) and therefore the classical paradox of the infinite velocity is avoided. The right-hand terms represent several effects: the first classical heat conduction (Fourier), the second Joule, the third Thomson effect and the last a dissipation related to the hyperbolic model.

Figure 1 schematizes the energy balance of the thermodynamics formulation. Electric and thermal energies are represented left and right, respectively; sources top and the flux bottom. The electric energy is irreversibly converted into thermal by the Joule effect, therefore the related arrow points towards the thermal energy side. Inside the domain, electric energy is reversibly converted into thermal and vice versa by three separated effects: Seebeck (thermal into electric), Peltier (electric into thermal) and Thomson in both ways.

\section{FEM formulation}

The aim of this section is to develop a variational formulation within the FE framework to permit the implementation of the thermoelectric problem into a computer code. For this purpose, consider a thermoelectric body of domain $\Omega$ and boundary $\Gamma$ governed by balance and transport equations obtained in the previous sections. In order to avoid a mixed Finite Element Method (FEM) formulation, the hyperbolic part $\tau \dot{\boldsymbol{S}}$ from the thermal transport equation (10)-bottom is introduced in the energy balance (4)-right, and the expression of the pure parabolic heat flux $\boldsymbol{q}$ is recovered

$$
\begin{aligned}
& \tau \rho c \ddot{T}+\rho c \dot{T}=-\nabla \cdot \boldsymbol{q}-\boldsymbol{j} \cdot \nabla V-\tau \partial_{t}\{\boldsymbol{j} \cdot \nabla V\} \\
& \boldsymbol{q}=-\kappa \nabla T+\alpha T \boldsymbol{j}
\end{aligned}
$$

The governing equations are complemented by Dirichlet (on boundaries $\Gamma_{V}, \Gamma_{T}$ ) and Neumann (on $\Gamma_{j}, \Gamma_{q}$ ) boundary conditions for electric and thermal fields

$$
\begin{array}{llll}
V=\bar{V} & \text { on } \Gamma_{V} ; & \boldsymbol{j} \cdot \mathbf{n}=j_{c} & \text { on } \Gamma_{j} \\
T=\bar{T} & \text { on } \Gamma_{T} ; & \boldsymbol{q} \cdot \mathbf{n}=q_{c} & \text { on } \Gamma_{q}
\end{array}
$$


where $\bar{V}, \bar{T}, j_{c}$ and $q_{c}$ are prescribed voltage, temperature, electric flux and thermal flux, respectively, and the outward normal is denoted by $\mathbf{n}$. Boundaries satisfy the conditions $\Gamma_{V} \cup \Gamma_{j}=\Gamma$, $\Gamma_{V} \cap \Gamma_{j}=0$ and $\Gamma_{T} \cup \Gamma_{q}=\Gamma, \Gamma_{T} \cap \Gamma_{q}=0$.

\subsection{Weak forms}

According to standard variational methods [22], the weak forms are obtained by multiplying the balance equations (4)left and (15)-top by variations of the degrees of freedom $\delta \mathrm{V}$, $\delta T$ and integrating over $\Omega$

$$
\begin{aligned}
& \int_{\Omega} \delta V \nabla \cdot \boldsymbol{j} \mathrm{d} \Omega=0 \\
& \int_{\Omega} \delta T \nabla \cdot \boldsymbol{q} \mathrm{d} \Omega+\int_{\Omega} \delta T \boldsymbol{j} \cdot \nabla V \mathrm{~d} \Omega+\int_{\Omega} \delta T \rho c \dot{T} \mathrm{~d} \Omega \\
& +\int_{\Omega} \delta T \tau \partial_{t}\{\boldsymbol{j} \cdot \nabla V\} \mathrm{d} \Omega+\int_{\Omega} \delta T \tau \rho c \ddot{T} \mathrm{~d} \Omega=0
\end{aligned}
$$

The $\tau$ and $\rho$-terms are added to the formulation developed in [15]. The divergence theorem is now applied to the first term of both equations, and 0 boundary conditions are introduced.

$$
\begin{aligned}
& -\int_{\Omega} \nabla \delta V \cdot \boldsymbol{j} \mathrm{d} \Omega+\int_{\Gamma} \delta V j_{c} \mathrm{~d} \Gamma=0 \\
& -\int_{\Omega} \nabla \delta T \cdot \boldsymbol{q} \mathrm{d} \Omega+\int_{\Omega} \delta T \boldsymbol{j} \cdot \nabla V \mathrm{~d} \Omega+ \\
& \int_{\Omega} \delta T \tau \partial_{t}\{\boldsymbol{j} \cdot \nabla V\} \mathrm{d} \Omega+\int_{\Omega} \delta T \tau \rho c \ddot{T} \mathrm{~d} \Omega+ \\
& \int_{\Omega} \delta T \rho c \dot{T} \mathrm{~d} \Omega+\int_{\Gamma} \delta T q_{c} \mathrm{~d} \Gamma=0
\end{aligned}
$$

\subsection{Discretization}

The continuum domain $\Omega$ is discretized with elements $\Omega_{e}$, see Figure 2. Therefore, $\Omega=\cup_{e=i}^{n_{e l}} \Omega_{e}$ where $n_{e l}$ is the total number of elements, each one delimited by $n_{p e}=8$ nodes. In turn, each node relates to two degrees of freedom (voltage, temperature) per node. For an element $e$, degrees of freedom and spatial coordinates $\mathbf{x}$ are approximated using 3-D isoparametric shape functions $\mathbf{N}_{e}$

$$
\begin{array}{llll}
V \approx V^{h} & =\mathbf{N}_{e} \mathbf{V}_{e} ; & T \approx T^{h} & =\mathbf{N}_{e} \mathbf{T}_{e} \\
\nabla V \approx \nabla V^{h} & =\mathbf{B}_{e} \mathbf{V}_{e} ; & & \nabla T \approx \nabla T^{h}=\mathbf{B}_{e} \mathbf{T}_{e} \\
\dot{T} \approx \dot{T}^{h} & =\mathbf{N}_{e} \dot{\mathbf{T}}_{e} ; & \ddot{T} \approx \ddot{T}^{h}=\mathbf{N}_{e} \ddot{\mathbf{T}}_{e} \\
\mathbf{x} \approx \mathbf{x}^{h} \quad=\mathbf{N}_{e} \mathbf{x}_{e} & &
\end{array}
$$

where $\mathbf{V}_{e}=\left\{V_{e}^{1}, \ldots, V_{e}^{n_{p e}}\right\}, \mathbf{T}_{e}=\left\{T_{e}^{1}, \ldots, T_{e}^{n_{p e}}\right\}$ and $\mathbf{B}_{e}$ is the discretized gradient matrix of the element

$$
\mathbf{B}_{A}^{t}=\left[\begin{array}{lll}
\frac{\partial N_{A}}{\partial x_{1}} & \frac{\partial N_{A}}{\partial x_{2}} & \frac{\partial N_{A}}{\partial x_{3}}
\end{array}\right]
$$

where $(\cdot)^{t}$ denotes matrix transpose. Similarly, the variations are discretized as

$$
\begin{array}{lll}
\delta V \approx \delta V^{h}=\mathbf{N}_{e} \delta \mathbf{V}_{e} ; & & \delta T \approx \delta T^{h}=\mathbf{N}_{e} \delta \mathbf{T}_{e} \\
\nabla \delta V \approx \nabla \delta V^{h}=\mathbf{B}_{e} \delta \mathbf{V}_{e} ; & & \nabla \delta \tau \approx \nabla \delta T^{h}=\mathbf{B}_{e} \delta \mathbf{T}_{e}
\end{array}
$$

Introducing these discretizations into the transport equations (10)-top and (15)-bottom

$$
\begin{aligned}
& \boldsymbol{j}_{e}=-\gamma \mathbf{B}_{e} \mathbf{V}_{e}-\alpha \gamma \mathbf{B}_{e} \mathbf{T}_{e} \\
& \boldsymbol{q}_{e}=-\kappa \mathbf{B}_{e} \mathbf{T}_{e}-\alpha \gamma \mathbf{N}_{e} \mathbf{T}_{e} \mathbf{B}_{e} \mathbf{V}_{e}-\alpha^{2} \gamma \mathbf{N}_{e} \mathbf{B}_{e} \mathbf{T}_{e}^{2}
\end{aligned}
$$

Introducing now (18) to (20) into (17) the Galerkin forms are obtained

$$
\begin{aligned}
& -\int_{\Omega_{e}} \mathbf{B}_{e}^{t} j_{e} \mathrm{~d} \Omega_{e}+\int_{\Gamma_{e}} \mathbf{N}_{e}^{t} j_{c} \mathrm{~d} \Gamma_{e}=0 \\
& -\int_{\Omega_{e}} \mathbf{B}_{e}^{t} \boldsymbol{q}_{e} \mathrm{~d} \Omega_{e}+\int_{\Omega_{e}} \mathbf{N}_{e}^{t} j_{e} \mathbf{B}_{e} \mathbf{V}_{e} \mathrm{~d} \Omega_{e}+ \\
& \int_{\Omega_{e}}^{\tau} \mathbf{N}_{e}^{t} \partial_{t}\left\{\boldsymbol{j}_{e} \mathbf{B}_{e} \mathbf{V}_{e}\right\} \mathrm{d} \Omega_{e}+\int_{\Omega_{e}} \tau \mathbf{N}_{e}^{t} \rho c \mathbf{N}_{e} \ddot{\mathbf{T}}_{e} \mathrm{~d} \Omega_{e} \\
& +\int_{\Omega_{e}} \mathbf{N}_{e}^{t} \rho c \mathbf{N}_{e} \dot{\mathbf{T}}_{e} \mathrm{~d} \Omega_{e}+\int_{\Gamma_{e}} \mathbf{N}_{e}^{t} q_{c} \mathrm{~d} \Gamma_{e}=0
\end{aligned}
$$

\subsection{Non-linear transient solution form}

In the present work a non-linear, transient hyperbolic problem is studied. The non-linearities are due to: (i) Joule heating, (ii) material dependence on temperature and (iii) coupling between electric and thermal fluxes. The transient behavior is relevant only for the thermal field, since $\rho_{\Omega}$ in (1) is assumed to be zero.

In order to solve the transient problem, the time interval of interest is divided into small increments, $\Delta t$, and the $\mathrm{N}-\beta$ or HHT method is introduced to replace time derivatives by discrete forms. Then for each time point the Newton-Raphson method is used to solve the resulting non-linear algebraic problem. These transient algorithms have been widely used in structural and solid mechanics (see [22]) and will not be described in detail here. Basically, the assembled non-linear FE equations are written in a residual form $\mathbf{R}$ and linearized by

$$
\mathbf{R}_{A}^{k}=-\left.\frac{\partial \mathbf{R}_{A}}{\mathbf{g}_{B}}\right|^{k} \mathrm{~d} \mathbf{g}_{A}^{k}
$$

where $A, B$ are the global numbering of two nodes, $k$ the Newton-Raphson iteration counter and $\mathbf{g}_{A}$ derivatives of the

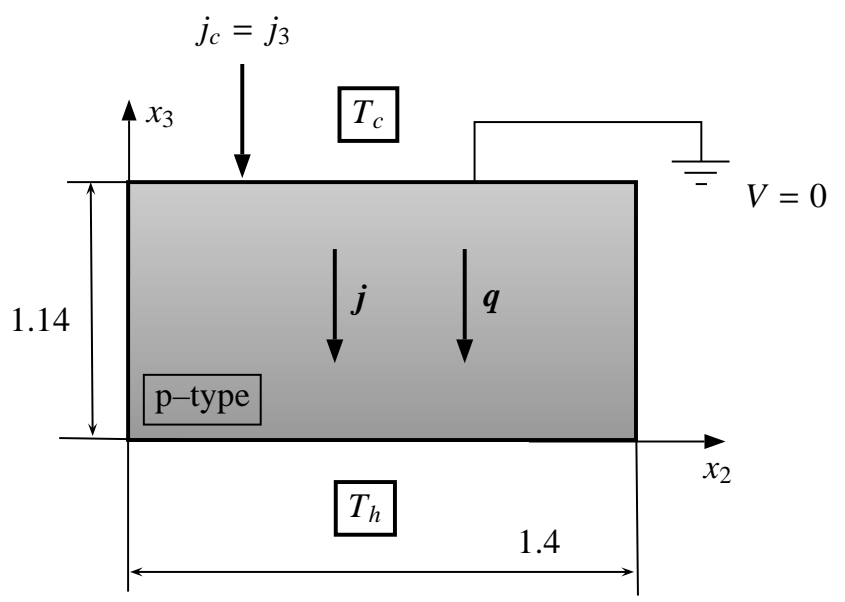

Figure 3: Thermoelement geometry and applied boundary conditions. 
degrees of freedom at node $A$ : value as $\mathbf{U}_{A}=\left\{V_{A}, T_{A}\right\}^{t}$, first derivative as $\dot{\mathbf{U}}_{A}=\left\{\dot{V}_{A}, \dot{T}_{A}\right\}^{t}$, and second derivative as $\ddot{\mathbf{U}}_{A}=$ $\left\{0, \ddot{T}_{A}\right\}^{t}$. The algorithms for time integration are taken as

$$
-\left.\frac{\partial \mathbf{R}_{A}}{\partial \mathbf{g}_{B}}\right|^{k}=c_{1} \mathbf{K}_{A B}+c_{2} \mathbf{C}_{A B}+c_{3} \mathbf{M}_{A B}
$$

The parameters $c_{1}, c_{2}$ and $c_{3}$ are given in Table 1 in terms of $\bar{\beta}, \bar{\gamma}$ for the $\mathrm{N}-\beta$ algorithm and in addition $\bar{\alpha}$ for the HHT algorithm.

\begin{tabular}{|l|c|c|c|}
\hline & \multicolumn{3}{|c|}{ Parameters } \\
\hline \hline Method & $c_{1}$ & $c_{2}$ & $c_{3}$ \\
\hline N- $\beta$ & 1 & $\frac{\bar{\gamma}}{\bar{\beta} \Delta t}$ & $\frac{1}{\bar{\beta} \Delta t^{2}}$ \\
\hline HHT & $\bar{\alpha}$ & $\frac{\bar{\alpha} \bar{\gamma}}{\bar{\beta} \Delta t}$ & $\frac{1}{\bar{\beta} \Delta t^{2}}$ \\
\hline
\end{tabular}

Table 1: Tangent matrix parameters for Newmark and HHT algorithms.

The consistent tangent, capacity and mass matrices, are derived for each iteration

$$
\mathbf{K}_{A B}=-\frac{\partial \mathbf{R}_{A}}{\partial \mathbf{U}_{B}} ; \quad \mathbf{C}_{A B}=-\frac{\partial \mathbf{R}_{A}}{\partial \dot{\mathbf{U}}_{B}} ; \quad \mathbf{M}_{A B}=-\frac{\partial \mathbf{R}_{A}}{\partial \ddot{\mathbf{U}}_{B}}
$$

Finally, the solution is updated by $\mathbf{g}_{B}^{k+1}=\mathbf{g}_{B}^{k}+\mathrm{d} \mathbf{g}_{B}^{k}$. Note that to ensure a correct derivation of the tangent matrix, the NewtonRaphson should exhibit a quadratic asymptotic rate of convergence.

From (22), the assembled residuals for each field at each global node are formulated as

$$
\begin{aligned}
& R_{A}^{V}=-\int_{\Omega} \mathbf{B}_{A}^{t} \boldsymbol{j}_{A} \mathrm{~d} \Omega+\int_{\Gamma} N_{A} j_{c} \mathrm{~d} \Gamma \\
& R_{A}^{T}=-\int_{\Omega} \mathbf{B}_{A}^{t} \boldsymbol{q}_{A} \mathrm{~d} \Omega+\int_{\Omega} N_{A} \dot{j}_{A} \mathbf{B}_{A} V_{A} \mathrm{~d} \Omega+ \\
& \int_{\Omega} N_{A} \tau \partial_{t}\left\{\dot{j}_{A} \mathbf{B}_{A} V_{A}\right\} \mathrm{d} \Omega+\int_{\Omega} N_{A} \tau \rho c \ddot{T}_{A} \mathrm{~d} \Omega+ \\
& \int_{\Omega} N_{A} \rho c \dot{T}_{A} \mathrm{~d} \Omega+\int_{\Gamma} N_{A} q_{c} \mathrm{~d} \Gamma
\end{aligned}
$$

where $\mathbf{B}_{A}$ is the discretized gradient vector at node $A$. From (25)-left and (26), the tangent stiffness matrices are

$$
\begin{aligned}
& K_{A B}^{V V}=-\partial_{V_{B}}\left\{R_{A}^{V}\right\}=\int_{\Omega} \mathbf{B}_{A}^{t} \partial_{V_{B}}\{\boldsymbol{j}\} \mathrm{d} \Omega \\
& K_{A B}^{V T}=-\partial_{T_{B}}\left\{R_{A}^{V}\right\}=\int_{\Omega} \mathbf{B}_{A}^{t} \partial_{T_{B}}\{\boldsymbol{j}\} \mathrm{d} \Omega \\
& K_{A B}^{T V}=-\partial_{V_{B}}\left\{R_{A}^{T}\right\}=\int_{\Omega} \mathbf{B}_{A}^{t} \partial_{V_{B}}\{\boldsymbol{q}\} \mathrm{d} \Omega- \\
& \int_{\Omega} N_{A} \partial_{V_{B}}\{\boldsymbol{j}\} \mathbf{B}_{B} V_{B} \mathrm{~d} \Omega-\int_{\Omega} N_{A} \boldsymbol{j}_{A} \mathbf{B}_{B} \mathrm{~d} \Omega- \\
& \int_{\Omega} N_{A} \tau \partial_{V_{B}}\{\boldsymbol{j}\} \mathbf{B}_{B} \dot{V}_{B} \mathrm{~d} \Omega-\int_{\Omega} N_{A} \tau \partial_{t}\{\boldsymbol{j}\} \mathbf{B}_{B} \mathrm{~d} \Omega \\
& K_{A B}^{T T}=-\partial_{T_{B}}\left\{R_{A}^{T}\right\}=\int_{\Omega} \mathbf{B}_{A}^{t} \partial_{T_{B}}\{\boldsymbol{q}\} \mathrm{d} \Omega- \\
& \int_{\Omega} N_{A} \partial_{T_{B}}\{\boldsymbol{j}\} \mathbf{B}_{B} V_{B} \mathrm{~d} \Omega-\int_{\Omega} N_{A} \tau \partial_{T_{B}}\{\boldsymbol{j}\} \mathbf{B}_{B} \dot{V}_{B} \mathrm{~d} \Omega
\end{aligned}
$$

Similar tangent matrices were obtained in [15] for the steadystate problem, for which both theories (parabolic and hyperbolic) are equivalent. Using (21) and the chain rule

$$
\begin{aligned}
\partial_{V_{B}}\{\boldsymbol{j}\} & =-\gamma \mathbf{B}_{B} \\
\partial_{T_{B}}\{\boldsymbol{j}\} & =-\alpha \gamma \mathbf{B}_{B}-\partial_{T}\{\alpha\} N_{B} \gamma \mathbf{B}_{B} T_{B} \\
& -\partial_{T}\{\gamma\}\left(\mathbf{B}_{B} V_{B}+\alpha \mathbf{B}_{B} T_{B}\right) N_{B} \\
\partial_{V_{B}}\{\boldsymbol{q}\} & =\alpha N_{B} T_{B} \partial_{V_{B}}\{\boldsymbol{j}\} \\
\partial_{T_{B}}\{\boldsymbol{q}\} & =\alpha N_{B} T_{B} \partial_{T_{B}}\{\boldsymbol{j}\}+\alpha \boldsymbol{j}_{B} N_{B}-\kappa \mathbf{B}_{B} \\
& +\partial_{T}\{\alpha\} \mathbf{N}_{B} T_{B} \boldsymbol{j}_{B} \mathbf{N}_{B}-\partial_{T}\{k\} \mathbf{B}_{B} T_{B} \mathbf{N}_{B}
\end{aligned}
$$

From (25)-middle and (26), the capacity matrices are

$$
\begin{aligned}
C_{A B}^{V V} & =-\partial_{\dot{V}_{B}}\left\{R_{A}^{V}\right\}=0 ; \quad C_{A B}^{V T}=-\partial_{\dot{T}_{B}}\left\{R_{A}^{V}\right\}=0 \\
C_{A B}^{T V} & =-\partial_{\dot{V}_{B}}\left\{R_{A}^{T}\right\}=-\tau \int_{\Omega} N_{A} \partial_{t \dot{V}_{B}}\{\boldsymbol{j}\} \mathbf{B}_{B} V_{B} \mathrm{~d} \Omega \\
& -\tau \int_{\Omega} N_{A} \boldsymbol{j}_{B} \mathbf{B}_{B} \mathrm{~d} \Omega \\
C_{A B}^{T T} & =-\partial_{\dot{T}_{B}}\left\{R_{A}^{T}\right\}=-\tau \int_{\Omega} N_{A} \partial_{t \dot{T}_{B}}\{\boldsymbol{j}\} \mathbf{B}_{B} V_{B} \mathrm{~d} \Omega \\
& -\int_{\Omega} N_{A} \rho c N_{B} \mathrm{~d} \Omega
\end{aligned}
$$

where the derivatives are obtained applying the chain rule to the discretized transport equations (21)

$$
\partial_{t \dot{V}_{B}}\{\boldsymbol{j}\}=-\gamma \mathbf{B}_{B} ; \quad \partial_{t \dot{T}_{B}}\{\boldsymbol{j}\}=-\alpha \gamma \mathbf{B}_{B}
$$

Again the symbol $\partial_{t \dot{V}_{B}}$ implies second partial derivative with respect to the time and the temporal derivative of the voltage. From (25)-right and (26), the mass matrices are obtained by

$$
\begin{aligned}
& M_{A B}^{V V}=-\partial_{\ddot{V}_{B}}\left\{R_{A}^{V}\right\}=0 ; \quad M_{A B}^{V T}=-\partial_{\ddot{T}_{B}}\left\{R_{A}^{V}\right\}=0 \\
& M_{A B}^{T V}=-\partial_{\ddot{V}_{B}}\left\{R_{A}^{T}\right\}=0 \\
& M_{A B}^{T T}=-\partial_{\ddot{T}_{B}}\left\{R_{A}^{T}\right\}=-\int_{\Omega} N_{A} \tau \rho c N_{B} \mathrm{~d} \Omega
\end{aligned}
$$

The final assembled matrix problem is given by

$$
\begin{aligned}
& {\left[\begin{array}{cc}
c_{1} \mathbf{K}^{T T}+c_{2} \mathbf{C}^{T T}+c_{3} \mathbf{M}^{T T} & c_{1} \mathbf{K}^{T V}+c_{2} \mathbf{C}^{T V} \\
c_{1} \mathbf{K}^{V T} & c_{1} \mathbf{K}^{V V}
\end{array}\right]\left\{\begin{array}{l}
\mathrm{d} \mathbf{T} \\
\mathrm{d} \mathbf{V}
\end{array}\right\}^{k}=\left\{\begin{array}{l}
\mathbf{R}^{T} \\
\mathbf{R}^{V}
\end{array}\right\}^{k}} \\
& \left\{\begin{array}{l}
\mathbf{T} \\
\mathbf{V}
\end{array}\right\}^{k+1}=\left\{\begin{array}{l}
\mathbf{T} \\
\mathbf{V}
\end{array}\right\}^{k}+\left\{\begin{array}{l}
\mathrm{d} \mathbf{T} \\
\mathrm{d} \mathbf{V}
\end{array}\right\}^{k}
\end{aligned}
$$

The present formulation is implemented as a user element in the non-linear finite element code FEAP, see [16]. Each element uses a standard (in the FEM sense) eight-node isoparametric element with two degrees of freedom per node: voltage $V_{A}$ and temperature $T_{A}$. 

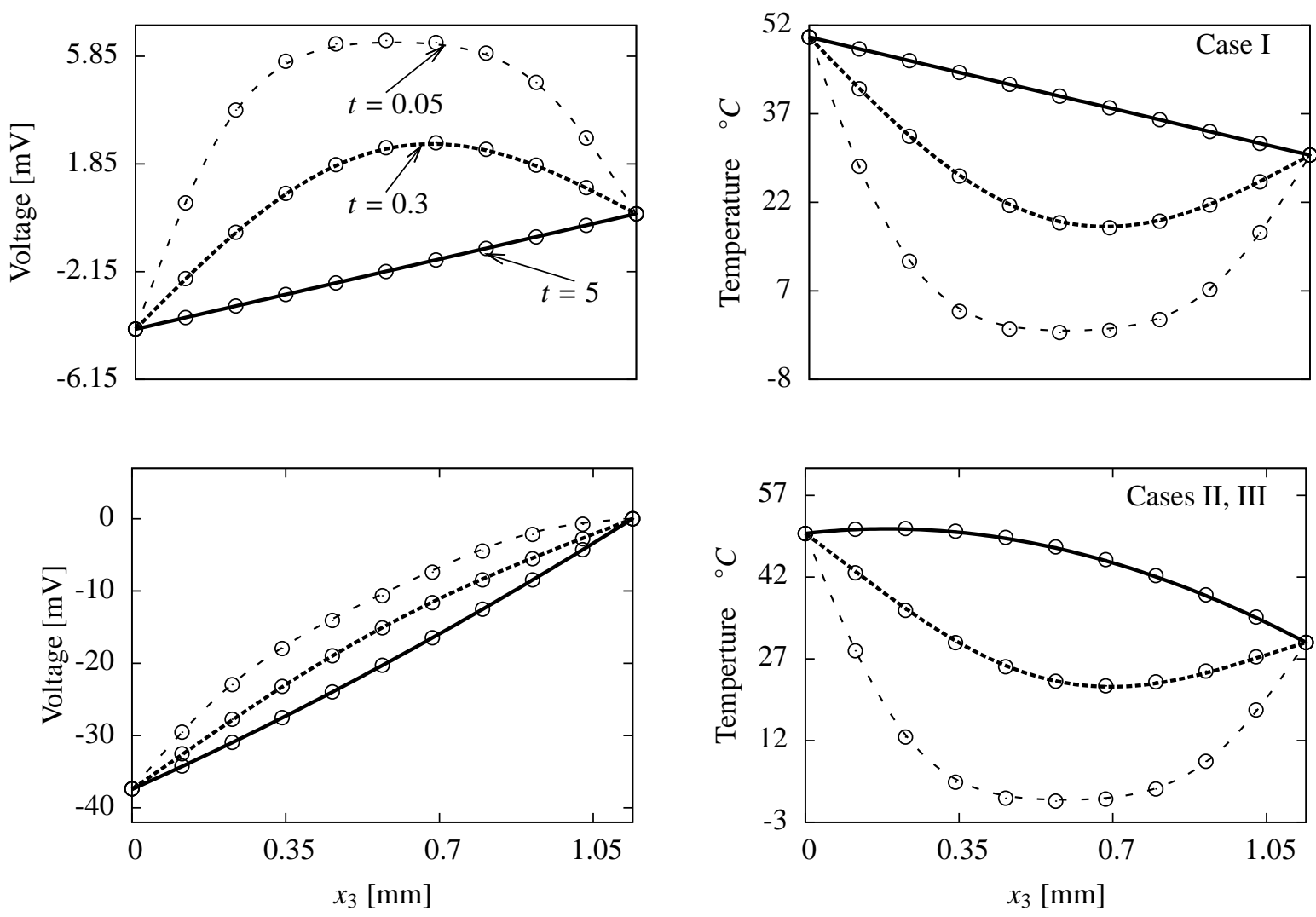

Figure 4: Voltage (left) and temperature (right) distributions along thermoelement for cases I (top row) and II (bottom row) and for three time instants. Analytical results with different line types, finite element with circles.
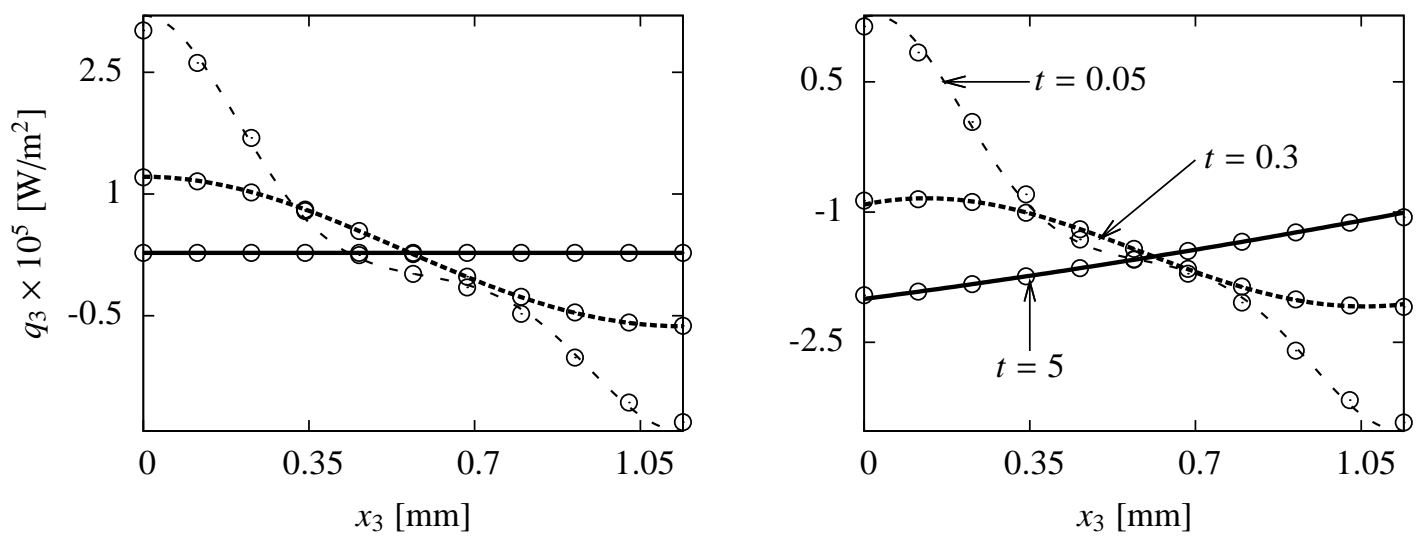

Figure 5: Thermal fluxes in cases I (left) and II (right) and for three times. Analytical results represented with different line types, finite element with circles.

\section{Validations}

The aim of this section is to validate the FE developed in Section 3 by means of four cases for which analytical solutions are found in the literature, modified or developed. These solutions are 1-D simplifications of the coupled partial differential equations (13), (14) and are summarized in Table 2. Due to difficulty in developing analytical solutions, the temperature dependence of the electric and thermal conductivities is not included in the validations. However, for the steady-state problem, this depen- dence has been verified in [23] using experimental results.

For all cases, a p-type thermoelement device manufactured by MELCOR [24] with properties given in (11) is modeled. The thermoelement is a parallelepiped of dimensions $1.4 \times 1.4 \times 1.14$ [mm]. For a p-type thermoelement, the electric flux direction is co-linear with the heat flux. The boundary conditions are discontinuous: initially the temperature is $T=0$ everywhere. For $t>0$ the boundary temperatures are set to $T_{c}=30$ on the cold face and to $T_{h}=50^{\circ} \mathrm{C}$ on the hot face. The boundary voltage is set to $V=0[\mathrm{~V}]$ on the cold face. Figure 3 shows the 
geometric dimensions and the boundary conditions.

\begin{tabular}{|c|l|l|}
\hline Case & Simplifications & Effects \\
\hline \hline I & $\tau=0 ; j=0 ; \alpha, \gamma, \kappa=c t e$ & $\mathrm{~F}, \mathrm{~S}$ \\
\hline II & $\tau=0 ; j \neq 0 ; \alpha, \gamma, \kappa=c t e$ & $\mathrm{~F}, \mathrm{~S}, \mathrm{~J}$ \\
\hline III & $\tau=0 ; j \neq 0 ; \alpha(T) ; \gamma, \kappa=c t e$ & $\mathrm{~F}, \mathrm{~S}, \mathrm{~J}, \mathrm{Th}$ \\
\hline IV & $\tau \neq 0 ; j \neq 0 ; \alpha(T) ; \gamma, \kappa=c t e$ & $\mathrm{~F}, \mathrm{~S}, \mathrm{~J}, \mathrm{Th}, \mathrm{ss}$ \\
\hline
\end{tabular}

Table 2: 1-D validation cases, simplifications and included effects: F - Fourier, $\mathrm{S}$ - Seebeck, J - Joule, Th - Thomson, ss - second sound

For cases II, III and IV, an electric flux in the $x_{3}$ direction is prescribed using the special interface element developed by the authors in [23], see Figure 2. The applied intensity is 5.2 $[\mathrm{A}]$ is an average of the ones specified by the manufacturer and

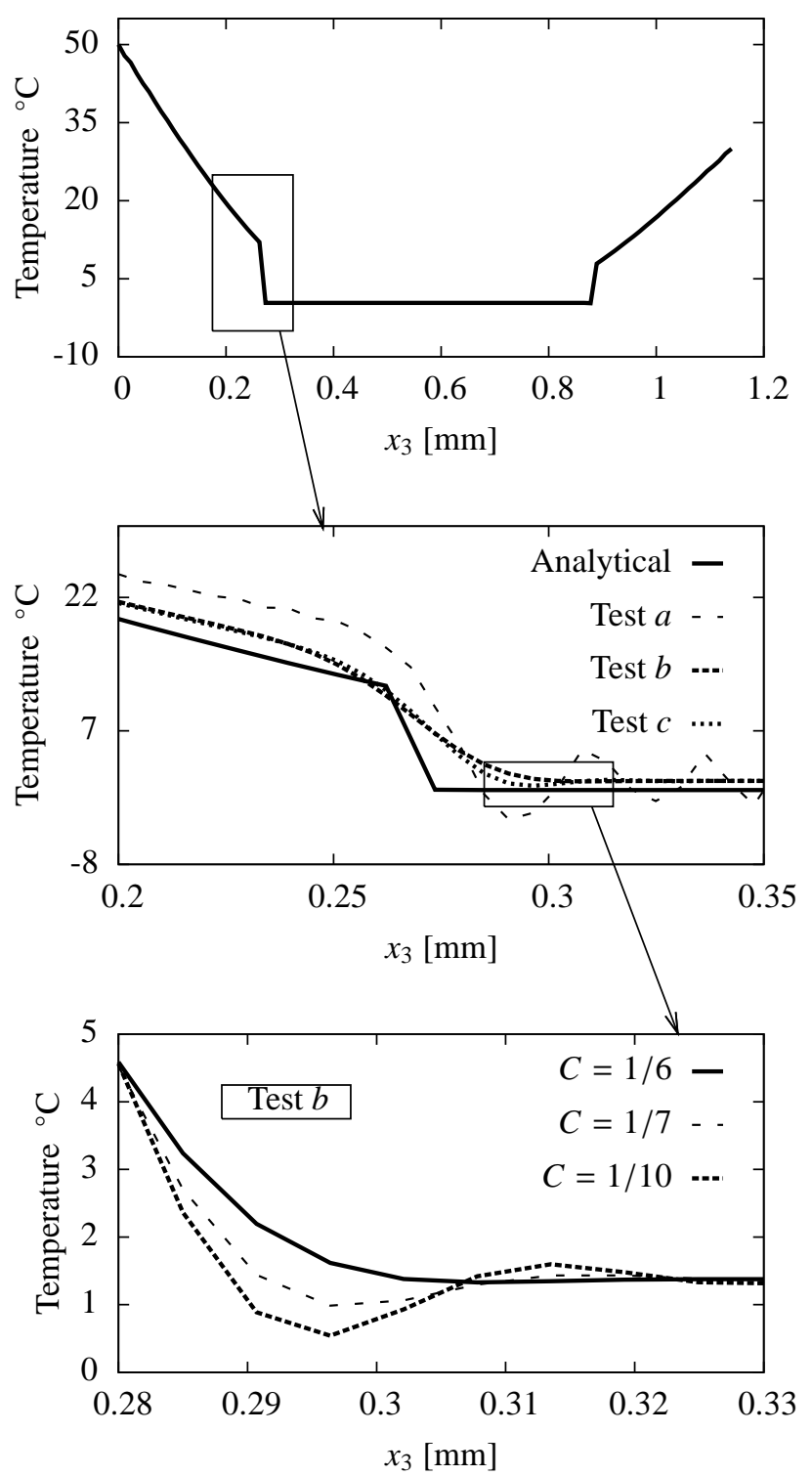

Figure 6: Analytical temperature solution for $t=0.06, \tau=0.02[\mathrm{~s}], C=1 / 6$ (top). N- $\beta$ and HHT parameters to study Gibbs phenomena (middle), see Table 3. Effect of the Courant number in Test $b$ by trial and error (bottom). corresponds to an electric flux $j_{3}=2.65 \times 10^{6}\left[\mathrm{~A} / \mathrm{m}^{2}\right]$.

The constant parameters for $\alpha, \gamma, \kappa$ are obtained from (11) using an average temperature $T_{m}=\left(T_{h}+T_{c}\right) / 2$.

For all cases, the electric strong form is directly given by (13), however, the thermal strong form for each case must be obtained by introducing the simplifications from Table 2 into (14).

$$
\begin{aligned}
& \text { I : } \quad \kappa \partial_{x x}\{T\}=\rho c \partial_{t}\{T\} \\
& \text { II : } \quad \kappa \partial_{x x}\{T\}=\rho c \partial_{t}\{T\}-j^{2} / \gamma \\
& \text { III : } \quad \kappa \partial_{x x}\{T\}=\rho c \partial_{t}\{T\}-j^{2} / \gamma+t_{h} j \partial_{x}\{T\} \\
& \text { IV : } \quad \kappa \partial_{x x}\{T\}=\rho c \partial_{t}\{T\}-j^{2} / \gamma+t_{h} j \partial_{x}\{T\}+ \\
& \\
& \tau \partial_{t}\left\{j \partial_{x}\{V\}\right\}+\tau \rho c \partial_{t t}\{T\}
\end{aligned}
$$

The fourth and fifth terms on the right side of case IV (second line) represent the second sound effects: irreversibility, (8)right, and hyperbolicity, respectively.

\subsection{Cases I to III}

For these cases, $\tau=0$ and the thermal strong forms (33) are diffusive: second order parabolic partial differential equations. For I, the situation is linear and homogeneous; for II linear and non-homogeneous and for III non-linear and nonhomogeneous.

Analytical solutions for the thermal field in cases I and II are given in [25], while the electrical fields can be calculated from (13), see the Appendix. For III, the thermal and electrical solutions are given in [26].

Numerical solutions are obtained using a structured (coarse) mesh of 11 elements in the $x_{3}$ direction. Only one element is used in the $x_{1}$ and $x_{2}$ directions since the problem is fundamentally $1-\mathrm{D}$. A time step of $\Delta t=0.1$ and the standard $\mathrm{N}-\beta$ parameters $\bar{\beta}=0.25, \bar{\gamma}=0.5$ are used.

Figure 4 shows the voltage (left) and temperature (right) distributions along the $x_{3}$ direction for case I (top), and for cases II and III (bottom). Solutions at $t=0.05,0.3,5$ [s] are represented: the analytical ones with lines and the FEM results with circles. For case I, the $T$ distributions are quadratic in nature during the initial transient response due to the boundary conditions. At near steady-state (e.g., $t=5$ ) they become nearly linear, since this problem is of the Laplace-type: the lack of electric current implies the absence of a Joule effect. The $V$ distributions are proportional to $T$, with $\alpha$ being the slope, see (13).

For case II, near quadratic distributions appear for the steady-state due to the Joule effect: this is a Poisson-type problem. Now, $V$ distributions are not proportional to $T$, since the potential drop increases due to electric energy being converted into thermal energy. Results for case III are very similar, since the Thomson effect is not relevant under the applied intensity, see [15]. As expected for these simple cases, the agreement between analytical and FEM distributions is very good.

Figure 5 shows the $x_{3}$ thermal flux vs. length. Analytical and FE results differ slightly at the edges, since the mesh is not 

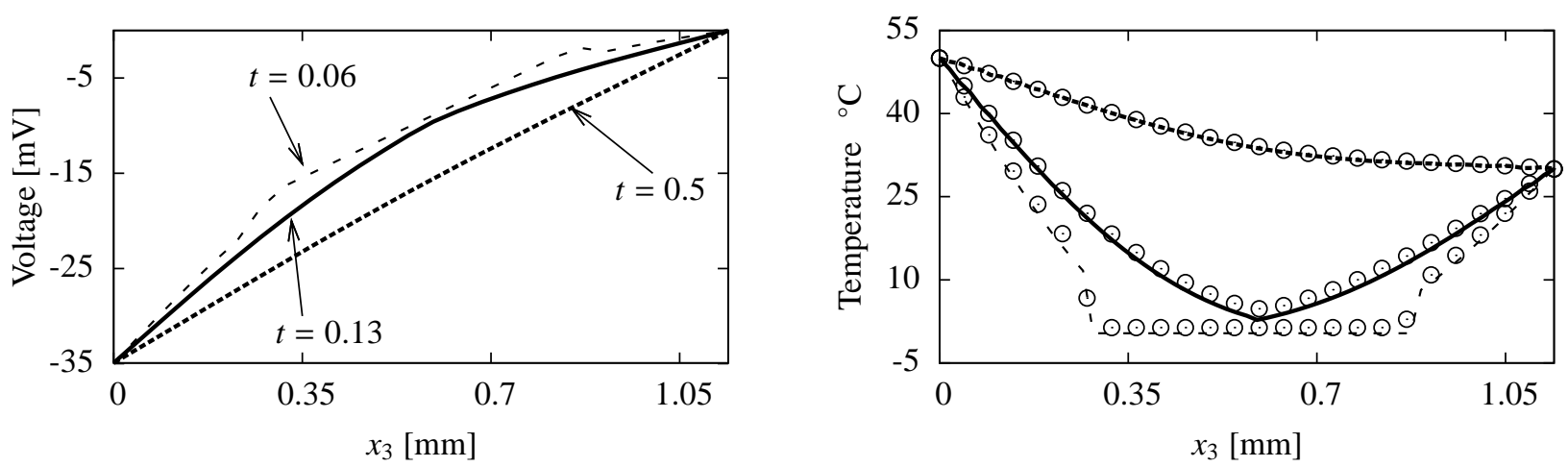

Figure 7: Voltage (left) and temperature (right) vs. thermoelement length at several time instants. For voltage, only finite element results. For temperature, analytical results are shown with different line types and corresponding finite element results with circles.

highly refined to capture the 0 boundary condition. For short times, the flux distributions are very similar in both cases, since the $T$ distributions have the same shape. Near steady-state, a constant distribution is obtained for case I (from the linear $T$ distribution) and a linear for case II (from a quadratic $T$ ). In addition, for case II the heat flux changes sign due to the second term in the right-face of (15)-bottom prevailing over the first one. Note that the prescribed electric flux is negative, according to the flux direction into the $\mathrm{p}$-type thermoelement. Again, case III is similar to case II and is not shown.

\subsection{Case IV}

Case IV is a non-linear and non-homogeneous hyperbolic problem. The analytical solution for the temperature is given in [13] and is based on a Laplace transform solution technique. However, several differences between this solution and our numerical results are found, thus, a new corrected comparison solution is deduced and summarized in the Appendix. Furthermore, in the present work the heat flux is calculated using a semi-analytical procedure that combines the analytical temperature solution with finite differences to obtain the gradient in (15)-bottom. No analytical results for voltage are generated due to the necessity of using numerical techniques in all steps.

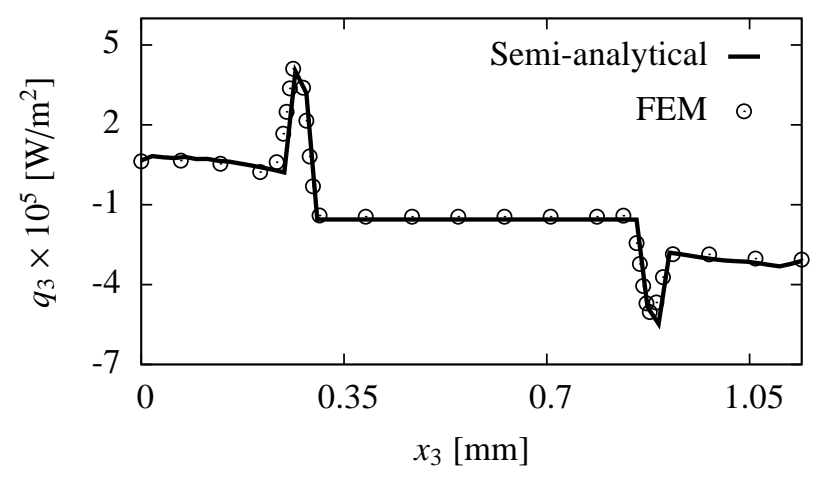

Figure 8: Thermal flux vs. thermoelement length for $t=0.06$. Semi-analytical result represented by solid lines and finite element by circles.
Differences between the parabolic and the hyperbolic models appear in the early times where the $V, T$ distributions appear as piecewise functions with sharp discontinuities near the edges. This occurs since both energies travel as finite velocity waves, see Figure 6. In addition, strong changes of temperature occur when discontinuous initial boundary conditions are used, inducing a Gibbs phenomena. For larger times, the parabolic model dominates and, therefore, the numerical algorithm must be:

- robust and efficient to automatically solve both parabolic and hyperbolic problems depending on the time instant.

- able to have controllable numerical dissipation to mitigate the Gibbs phenomena.

For a linear problem and according to [10], no numerical oscillations will appear if the dynamic algorithm is regularized by the relationship $\Delta t \leq C h / v$, where $C$ is the Courant number [27] and $v=\sqrt{\kappa / \rho c \tau}$, the linear wave velocity. The cases considered in the present work are highly non-linear, therefore the spectrum of the algorithm is not evident enough for a good estimation of the ratio $\Delta t / h$. In our analyses a structured mesh of 200 elements $\left(h=8.1429 \times 10^{-6}\right)$ in the $x_{3}$ direction is used. Since a precise spectral analysis is not an objective of this paper, a value $C=1 / 6$ is chosen selected based on a series of numerical tests. If smaller values of $C$ (e.g., 1/10 and consequently smaller $\Delta t$ ) are used the oscillation appear again, as seen in Figure 6 bottom. Larger values of $C$ will artificially smear the distribution at the front itself.

In order to evaluate the performance of the time integration scheme, $\mathrm{N}-\beta$ and HHT algorithms are compared using parameters that produce different spectral radii $\bar{\rho}$, see [28] and Table 3. According to [29], $\bar{\rho}$ should stay close to unity for small to intermediate time steps and decrease to $\bar{\rho} \approx 0.5$ only when $\Delta t / \hat{T} \rightarrow \infty$, where $\hat{T}$ is the undamped natural period.

Figure 6 top shows $T$ vs. length $x_{3}$ for an assumed $\tau=0.02$ (as in most of this section) at $t=0.06$; for shorter times the comparison analytical solution does not converge; the numerical one does although with increasing Gibbs noise. The analytical solution (solid line) and the FE (dashed) are obtained with 
$\mathrm{N}-\beta$ and HHT algorithms (these are unconditionally stable for the parameters tested when applied to linear problems).

\begin{tabular}{|c|c|l|l|l|l|}
\hline Test & Algorithm & $\bar{\rho}$ & $\bar{\beta}$ & $\bar{\gamma}$ & $\bar{\alpha}$ \\
\hline \hline$a$ & $\mathrm{~N}-\beta$ & 1 & $1 / 4$ & $1 / 2$ & - \\
\hline & $\mathrm{N}-\beta$ & $3 / 2$ & $9 / 14$ & $16 / 49$ & - \\
\hline & $\mathrm{N}-\beta$ & $1 / 2$ & $4 / 9$ & $5 / 6$ & - \\
\hline$b$ & $\mathrm{~N}-\beta$ & 0 & 1 & $3 / 2$ & - \\
\hline \hline & HHT & $3 / 2$ & $9 / 14$ & $16 / 49$ & $6 / 7$ \\
\hline$c$ & HHT & $1 / 2$ & $4 / 9$ & $5 / 6$ & $2 / 3$ \\
\hline
\end{tabular}

Table 3: Newmark- $\beta$ and HHT parameters used for numerical testing.

For $\mathrm{N}-\beta$, the use of standard parameters (test $a$ ) results in numerical oscillations since this algorithm is non-dissipative. The HHT algorithm (test $c$ ) slightly oscillates due to its low numerical damping. Therefore, in the remainder of the present work the parameters of test $b$ will be used, since according to [28] this choice results in the highest numerical dissipation.

Figure 7 shows the $V$ (left) and $T$ (right) distributions along the thermoelement at several instants of time. In the first, it can be observed the transition from very small times and hyperbolic solution to higher ones and parabolic, the last with almost linear distribution between boundary values. Notice in the curve for $t=0.06$ the electric wave fronts from left and right at around 0.28 and $0.8[\mathrm{~mm}]$, with a non-linear distribution inside the wave and linear between the fronts. For $t=0.13$ the both waves almost collide at around $0.63[\mathrm{~mm}]$, point shifted to the right. This is so since the $T$ boundary condition is higher in the left: the energy and consequently velocity are also higher in the left wave.

In the $T$ distribution a similar transition from hyperbolic to parabolic can be seen. The collision between the thermal waves is again clear for $t=0.13$ at the same thermoelement point, a logical result since the thermal and electric waves are coupled. For $t=0.06$ it can be appreciated that inside the wave zone the slope of the distribution is higher than the obtained with a parabolic model, with the consequence of a higher energy confined in a smaller volume. The agreement between analytical and numerical results is very good, except for a small region around the wave front at $t=0.06$; this is due to the intrinsic oscillations from the discrete time integration scheme and is very difficult to avoid

Figure 8 compares the semi-analytical and FEM thermal fluxes for $t=0.06$. Two peaks due to the discontinuous boundary conditions at the wave front are evident, representing the propagation of this wave. Even if a nil flux at the thermoelement center could be expected, it has a constant negative value. The reason is found in (15) bottom: the first right-term is zero due to a constant $T$ distribution where the wave is not present (see Figure 7), distribution that is due to the electric coupling, see the last term in (15) top. But the second term in (15) bottom will be negative due to the prescribed $j$. The agreement is again very good, even at the peak maximums, although it has to be considered that both maximums can be affected by the respective choices of the numerical parameters.

As mentioned before, the behavior is hyperbolic in the initial instants and becomes parabolic later. This is clearly seen in Figure 9 top, where the heat flux is plotted for several times. For $t<0.06$ the peaks described in the previous figure are visible, but for larger times they become smoother. At $t=0.13$ both waves collide, and after that the flux will be linear as in Figure 5. The mathematical explanation of this attenuation can be seen in (15)-top: the first term in the left hand side is hyperbolic, the second parabolic and the last in the right dispersive. In the initial instants the hyperbolic term is dominant; physically this can be interpreted as a ballistic motion of either electrons or holes. The wave is constantly attenuated by the dissipation introduced by the last term up to a time in which the influence of the $\tau$ terms vanish, and the first time derivative is the dominant term.

The last attenuation is obviously affected by the value of the constant $\tau$, see Figure 9 bottom, where it can be seen that for larger $\tau$ the behavior will be more hyperbolic (more difficult to simulate) and vice-versa. This figure is a test for the robustness of the finite element performance, but even for $\tau=0.06$ the simulation is satisfactory as long as the ratio $\Delta t \leq C h / v$ is used.

\section{Conclusions}

This article has presented a non-linear and transient finite element formulation to simulate thermoelectric behavior under hyperbolic heat conduction. The formulation has been implemented into the computational code FEAP. Numerically, nonlinearities and transient hyperbolicity have been addressed by Newton-Rhapson and by Newmark- $\beta$ and HHT algorithms, respectively. Analytically, four 1-D solutions have been obtained to validate the finite element results; the first three solve parabolic problems and both result types agree very well, using standard parameters of Newmark- $\beta$. The fourth validation consists on a hyperbolic problem with discontinuous initial boundary conditions. In this example, voltage and temperature distributions present sharp discontinuities, and the numerical results numerical oscillations. To mitigate this Gibbs phenomena three numerical test have been developed: Newmark $\beta$ with standard parameters, HHT and Newmark $-\beta$ with $\bar{\beta}=1, \bar{\gamma}=3 / 2$. The latter test provides the best results since this parametrization gives the highest numerical dissipation. In addition, the time steps and element sizes have been regularized using a linear relationship with a Courant number $C=1 / 6$. This $C$ has been chosen by means of trial and error, since the problem is highly non-linear and a spectral analysis is not one of the current work objectives. Physically, the obtained results show a wave propagation of temperature, of thermal flux and of voltage (due to the thermoelectric coupling). This numerical tool can be applied to the design of micro-devices for novel applications with fast processes, where the second sound effect could be relevant.

\section{Acknowledgments}

This research was partially supported by the spanish Ministry of Education through Grant No. FPU AP-2006-02372 and also from grants MICINN BIA-2008-00522, Excelencia Junta Andalucía P08-TEP-03641 and “Ayudas Investigación” from UPV 

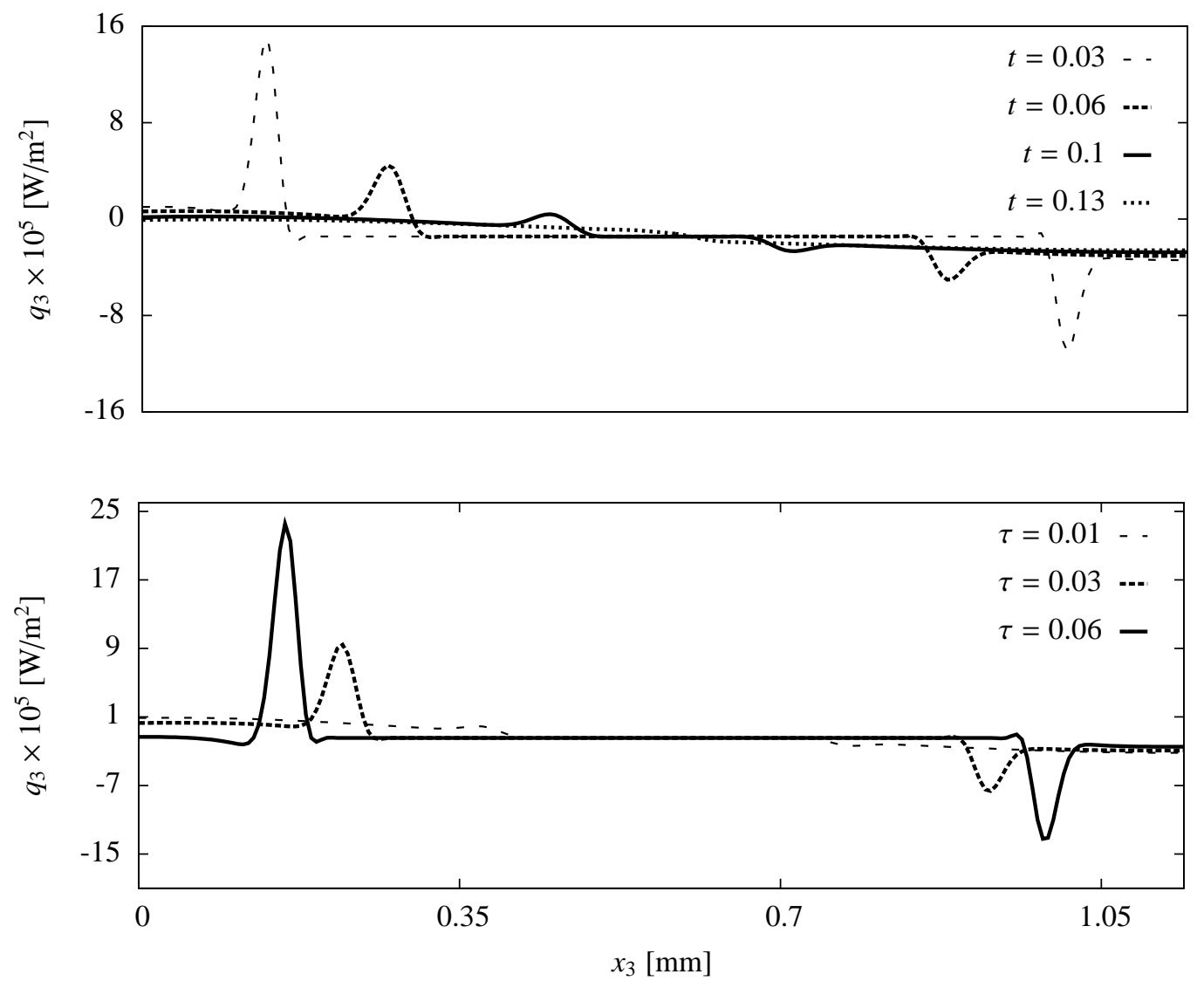

Figure 9: Thermal flux vs. thermoelement length at several time instants for $\tau=0.02$ (top) and relaxation time influence in the wave shape (bottom) at $t=0.06$. Only finite element results represented.

grants. The authors would also like to thank Prof. Guillermo Rus for his valuable contributions.

\section{References}

[1] S. Riffat, X. Ma, Thermoelectrics: a review of present and potential applications, Applied Thermal Engineering 23 (2003) 913-935.

[2] G. Snyder, J. Fleurial, T. Caillat, Supercooling of Peltier cooler using a current pulse, Journal of Applied Physics 92 (3) (2002) 1564-1569.

[3] C. Cattaneo, Sulla conduzione del calore, Atti Seminario Mat. Fis. University Modena 3 (1938) 83-101.

[4] D. Jou, G. Lebon, Extended Irreversible Thermodynamics, SpringerVerlag Berlin Heidelberg, 1996.

[5] G. Lebon, D. Jou, J. Casas-Vázquez, Understanding Non-equilibrium Thermodynamics, Springer-Verlag, 2008.

[6] J. Gembarovic, V. Majernik, Non-fourier propagation of heat pulses in finite medium, International Journal of Heat and Mass Transfer 31 (5) (1988) 1073-1080.

[7] H. Chen, J. Lin, Numerical analysis for hyperbolic heat conduction, International Journal of Heat and Mass Transfer 36 (11) (1993) 2891-2898.

[8] A. Barletta, E. Zanchini, Hyperbolic heat conduction and local equilibrium: a second law analysis, International Journal of Heat and Mass Transfer 40 (5) (1997) 1007-1016.

[9] M. Manzari, M. Manzari, On numerical solution of hyperbolic heat conduction, Communications in Numerical Methods in Engineering 15 (1999) 853-866.

[10] R. Ciegis, Numerical solution of hyperbolic heat conduction equation, Mathematical modelling and analysis 14 (1) (2009) 11-24.

[11] H. Gómez, I. Colominas, F. Navarrina, M. Casteleiro, A discontinuous Galerkin method for a hyperbolic model for convection-diffusion prob- lems in CFD, International Journal for Numerical Methods in Engineering 71 (2007) 1342-1364.

[12] N. Yu, S. Imatani, T. Inoue, Hyperbolic thermoelastic analysis due to pulsed heat input by numerical solution, JSME International Journal Series A 49 (2) (2006) 180-187.

[13] M. Alata, M. Al-Nimr, M. Naji, Transient behaviour of a thermoelectric device under the hyperbolic heat conduction model, International Journal of Thermophysics 24 (6) (2003) 1753-1768.

[14] E. Antonova, D. Looman, Finite elements for thermoelectric device analysis in ANSYS, in: International Conference on Thermoelectrics, 2005.

[15] J. Pérez-Aparicio, R. Taylor, D. Gavela, Finite element analysis of nonlinear fully coupled thermoelectric materials, Computational Mechanics 40 (2007) 35-45.

[16] R. Taylor, FEAP A Finite Element Analysis Program: User Manual, University of California, Berkeley, http://www.ce.berkeley.edu/feap (2010).

[17] H. Hilber, T. Hughes, R. Taylor, Improved numerical dissipation for time integration algorithms in structural dynamics, Earthquake Engineering and Structural Dynamics 5 (1977) 282-292.

[18] C. Truesdell, Rational Thermodynamics, Springer, Berlin Heidelberg New York, 1984.

[19] J. Jackson, Classical Electrodynamics, John Wiley and Sons, Inc., 1962.

[20] A. Eringen, G. Maugin, Electrodynamics of Continua I, Springer-Verlag New York, Inc., 1990.

[21] A. Chakraborty, K. Ng, Thermodynamic formulation of temperatureentropy diagram for the transient operation of a pulsed thermoelectric cooler, International Journal of Heat and Mass Transfer 49 (2006) 18451850 .

[22] O. Zienkiewicz, R. Taylor, J. Zhu, The Finite Element Method: The Basis, Elsevier Butterworth-Heinemann, 2005.

[23] J. Pérez-Aparicio, R. Palma, R. Taylor, Finite element analysis and material sensitivity of Peltier thermoelectric cells coolers, International Journal 
of Heat and Mass Transfer (2010) Submitted.

[24] MELCOR, Application notes for thermoelectric devices, Tech. rep., Melcor: Laird Technologies, http://www.lairdtech.com.

[25] A. Polyanin, Handbook of Linear Partial Differential Equations for Engineers and Scientist, Chapman \& Hall CRC, 2002.

[26] W. Seiffert, M. Ueltzen, E. Muller, One-dimensional modelling of thermoelectric cooling, Phys. Stat. Sol. A 194 (1) (2002) 277-290.

[27] K. F. R. Courant, H. Lewy, Uber die partiellen differenzengleichungen der mathematischen physik, Mathematische Annalen 100 (2007) 32-74.

[28] T. Hughes, The Finite Element Method. Linear Static and Dynamic Finite Element Analysis, Prentice-Hall, Inc., 1987.

[29] T. Fung, Numerical dissipation in time-step integration algorithms for structural dynamic analysis, Progress in Structural Engineering and Materials 5 (2003) 167-180.

\section{Appendix A. Analytical solutions}

The analytical solution of case III is given in [26]. In this appendix specially developed solutions for I, II, IV are presented.

The temperature distribution along the thermoelement for I, II are reported in [25]. The voltage distributions are calculated by means of (13) and the thermal flux by (15)-bottom.

\section{Case I}

$$
\begin{aligned}
& V\left(x_{3}, t\right)=-\alpha\left(\frac{\Delta T}{L} x_{3}-\Delta T+\sum_{n=1}^{\infty} c_{n} \mathrm{Sx} \mathrm{e}^{-\Lambda t}\right) \\
& T\left(x_{3}, t\right)=T_{c}+\frac{\Delta T}{L} x_{3}+\sum_{n=1}^{\infty} c_{n} \mathrm{Sx} \mathrm{e}^{-\Lambda t} \\
& q_{3}\left(x_{3}, t\right)=-\kappa\left(\frac{\Delta T}{L}+\sum_{n=1}^{\infty} c_{n} \mathrm{Cx} \frac{n \pi}{L} \mathrm{e}^{-\Lambda t}\right)
\end{aligned}
$$

where $\Delta T=T_{h}-T_{c}, \Lambda=\beta(n \pi / L)^{2}, \beta=\kappa / \rho c, \mathrm{Sx} \equiv$ $\sin \left(n \pi x_{3} / L\right), \mathrm{Cx} \equiv \cos \left(n \pi x_{3} / L\right)$ and $L$ is the thermoelement length. In addition

$$
c_{n}=\frac{2}{L} \int_{0}^{L}\left(T_{c}-\frac{\Delta T}{L} x_{3}\right) \mathrm{Sxd} x_{3}
$$

\section{Case II}

$$
\begin{aligned}
V\left(x_{3}, t\right)= & \frac{j_{3}}{\gamma} \mathrm{Lx}+\alpha\left[\left(\frac{\Delta T}{L}-A_{1} x_{3}\right) \mathrm{Lx}\right. \\
& \left.-\sum_{n=1}^{\infty} c_{n} \mathrm{Sx} \mathrm{e}^{-\Lambda t}\right] \\
T\left(x_{3}, t\right)= & \frac{A_{2}}{L} x_{3}+T_{C}-A_{3} x_{3}^{2}+\sum_{n=1}^{\infty} c_{n} \mathrm{Sx}^{-\Lambda t} \\
q_{3}\left(x_{3}, t\right)= & -\kappa\left(\frac{A_{2}}{L}-2 A_{3} x_{3}+\sum_{n=1}^{\infty} c_{n} \mathrm{Cx} \frac{n \pi}{L} \mathrm{e}^{-\Lambda t}\right) \\
& +\alpha T\left(x_{3}, t\right) j_{3}
\end{aligned}
$$

where Lx $=L-x_{3}, A_{1}=j_{3} / 2 \kappa \gamma, A_{2}=\Delta T+j_{3}^{2} L^{2} / 2 \beta \rho c \gamma$, $A_{3}=j_{3}^{2} / 2 \beta \rho c \gamma$ and

$$
c_{n}=\frac{2}{L} \int_{0}^{L}\left(\frac{A_{2}}{L} x_{3}+T_{c}-A_{3} x_{3}^{2}\right) \mathrm{Sx} \mathrm{d} x_{3}
$$

\section{Case IV}

The temperature distribution along the thermoelement was reported in [13] using the Laplace transform technique. However, we believe there are several errors in this work, for which the result is developed again. From the reference, we use the dimensionless parameters $\xi=x_{3} / L$ and $\eta=t \kappa / \rho c L^{2}$ but (14) is rewritten in an amenable form to be solved by the Laplace technique

$$
\begin{aligned}
& \partial_{\xi \xi}\{T\}+F_{1} \partial_{\xi}\{T\}+F_{2}-\partial_{\eta}\{T\}-\tau F_{3} \partial_{\eta \eta}\{T\}+ \\
& \tau F_{4} \partial_{\eta}\left\{F_{2} \frac{A \kappa}{L^{2}}-\frac{\alpha \kappa A}{t_{h} L^{2}} F_{1} \partial_{\xi}\{T\}\right\}=0 \\
& T(\xi, 0)=0 ; \quad T(0, \eta)=T_{h} ; \quad T(1, \eta)=T_{c}
\end{aligned}
$$

Note that the boundary conditions have been included. Regarding [13], $F_{1}=-t_{h} I L / \kappa A, F_{2}=I^{2} L^{2} / \kappa \gamma A^{2}$ have been modified and $F_{3}=\kappa / \rho c L^{2}, F_{4}=1 / \rho c A$ are added. Applying the Laplace transform

$$
\begin{aligned}
& W(\xi, s)=P_{11} \mathrm{e}^{\lambda_{11} \xi}+P_{12} \mathrm{e}^{\lambda_{12} \xi}+\frac{F_{2}}{s\left(\tau F_{3} s^{2}+s\right)} \\
& W(\xi)=0 ; \quad W(0)=\frac{T_{c}}{s} ; \quad W(1)=\frac{T_{h}}{s}
\end{aligned}
$$

where $s$ is time in the Laplace domain. The boundary conditions in (A.6) are different from those in [13]: in this reference they are not transformed. Then, the following parameters are modified

$$
\begin{aligned}
& P_{11}=\frac{\frac{T_{h}}{s}+\mathrm{e}^{\lambda_{12}}\left(\frac{F_{2}}{s\left(\tau F_{3} s^{2}+s\right)}-\frac{T_{c}}{s}\right)-\frac{F_{2}}{s\left(\tau F_{3} s^{2}+s\right)}}{\mathrm{e}^{\lambda_{11}}-\mathrm{e}^{\lambda_{12}}} \\
& P_{12}=\frac{T_{c}}{s}-P_{11}-\frac{F_{2}}{s\left(\tau F_{3} s^{2}+s\right)} \\
& \left\{\begin{array}{c}
\lambda_{11} \\
\lambda_{12}
\end{array}\right\}=\frac{1}{2}\left(-A_{1} \mp \sqrt{A_{1}^{2}-4 B_{1}}\right) \\
& A_{1}=F_{1}\left(t_{h} L^{2}+A \alpha \kappa \tau s F_{4}\right) ; \quad B_{1}=-t_{h}^{2} L^{4}\left(s+F_{3} s^{2} \tau\right)
\end{aligned}
$$

Finally, (A.6) are inverted using the Riemann-sum approximation as in [13]. 